\title{
First measurement of the hydrogen spin-exchange collision cross-section in the low temperature region
}

\author{
C. Baumgarten ${ }^{1, a}$, B. Braun ${ }^{2}$, M. Capiluppi ${ }^{3}$, G. Ciullo ${ }^{3}$, P.F. Dalpiaz ${ }^{3}$, H. Kolster ${ }^{1,4, b}$, P. Lenisa $^{3, c}$, H. Marukyan $^{5}$,

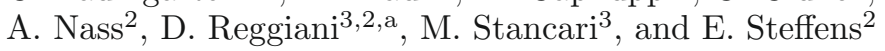 \\ 1 Sektion Physik, Ludwigs-Maximillians-Universität Mḍotunchen, 85748 Garching, Germany \\ 2 Physikalisches Institut Universität Erlangen-Nürnberg, 91058 Erlangen, Germany \\ 3 Dipartimento di Fisica Universita' di Ferrara, 44100 Ferrara, Italy \\ 4 NIKHEF, 1009 DB Amsterdam, The Netherlands \\ 5 Yerevan Physics Institute, 375036 Yerevan, Armenia
}

Received 20 March 2008 / Received in final form 23 May 2008

Published online 21 June 2008 - (C) EDP Sciences, Società Italiana di Fisica, Springer-Verlag 2008

\begin{abstract}
The spin-exchange collision cross-section for hydrogen atoms has been measured for the first time in the low temperature range $40-100 \mathrm{~K}$ by using the polarized hydrogen gas target of the HERMES experiment at DESY (Hamburg, Germany). The results agree with a previous measurement in the overlapping temperature region $80-100 \mathrm{~K}$, while seem to hint an increasing behaviour with temperature in the region $50-80 \mathrm{~K}$.
\end{abstract}

PACS. 39.10.+j Atomic and molecular beam sources and techniques $-13.88 .+\mathrm{e}$ Polarization in interactions and scattering

\section{Introduction}

The process of spin exchange occurring in the collision of two hydrogen atoms in the ground state is of interest in different fields of physics, e.g. in astrophysics [1-4], atomic physics [5-10] and in particular for the hydrogen maser $[8,10,11]$.

The hydrogen-hydrogen spin-exchange interaction and estimates of the corresponding cross-section have been considered in a number of theory papers $[1-3,5,7,8,12]$. Spin-exchange collisions are caused by the large difference in the interaction potentials for electronic spin singlet state $V_{s}$ and triplet state $V_{t}$. The method introduced by Purcell and Field [1] to calculate the cross-section for spinexchange collisions $\sigma_{s e}$ is based on the estimation of the phase shift difference $\phi_{t s}$ for these potentials

$$
\phi_{t s}=\int \frac{V_{t}-V_{s}}{\hbar} d t
$$

where the integral is taken over the time duration of the collision. The phase shift $\phi_{t s}$ depends on both the impact parameter $b$ and the energy of the incident particle.

\footnotetext{
a Present address: Paul Scherrer Institut, 5232 Villigen, Switzerland.

b Present address: Massachusetts General Hospital Department of Radiology, MGH Charlestown, MA 02129, USA.

${ }^{c}$ e-mail: lenisa@hermes.desy.de
}

At a given energy the total spin exchange cross-section is given by

$$
\sigma_{s e}=2 \pi \int_{0}^{\infty} \sin ^{2}\left(\frac{1}{2} \phi_{t s}\right) b d b=\pi \int_{0}^{\infty}\left(1-\cos \phi_{t s}\right) b d b .
$$

The first calculation of $\sigma_{s e}$ was done in 1956 [1] using the potential curves calculated by Hirschenfelder in 1954 [13]. A more recent calculation of the spin-exchange crosssection was performed by Swenson et al. [14] using different potential curves from [15].

The method introduced by Wittke and Dicke [5] makes use of partial wave treatment of the scattering process. The two outgoing waves of a given orbital angular momentum $l$ acquire a phase difference due to the difference in the potential between the triplet and singlet states and the cross-section of the spin-exchange collision may be written

$$
\sigma_{s e}=\frac{\pi}{k^{2}} \sum_{l=0}^{\infty}(2 l+1) \sin ^{2}\left(\delta_{l}^{s}-\delta_{l}^{t}\right),
$$

where $\delta_{l}^{s}$ and $\delta_{l}^{t}$ are the phase shifts for the singlet and triplet potentials and $k=\mu v_{r} / \hbar, \mu$ is the reduced mass of the atom, $v_{r}$ is the relative velocity of the hydrogen atoms in a collision.

One of the most recent and satisfactory calculations of the value of $\sigma_{s e}$ has been given by Allison [16], using the precise hydrogen-hydrogen interaction potential of Kolos and Wolniewicz [17]. Depending on the total parity $+/-$ 


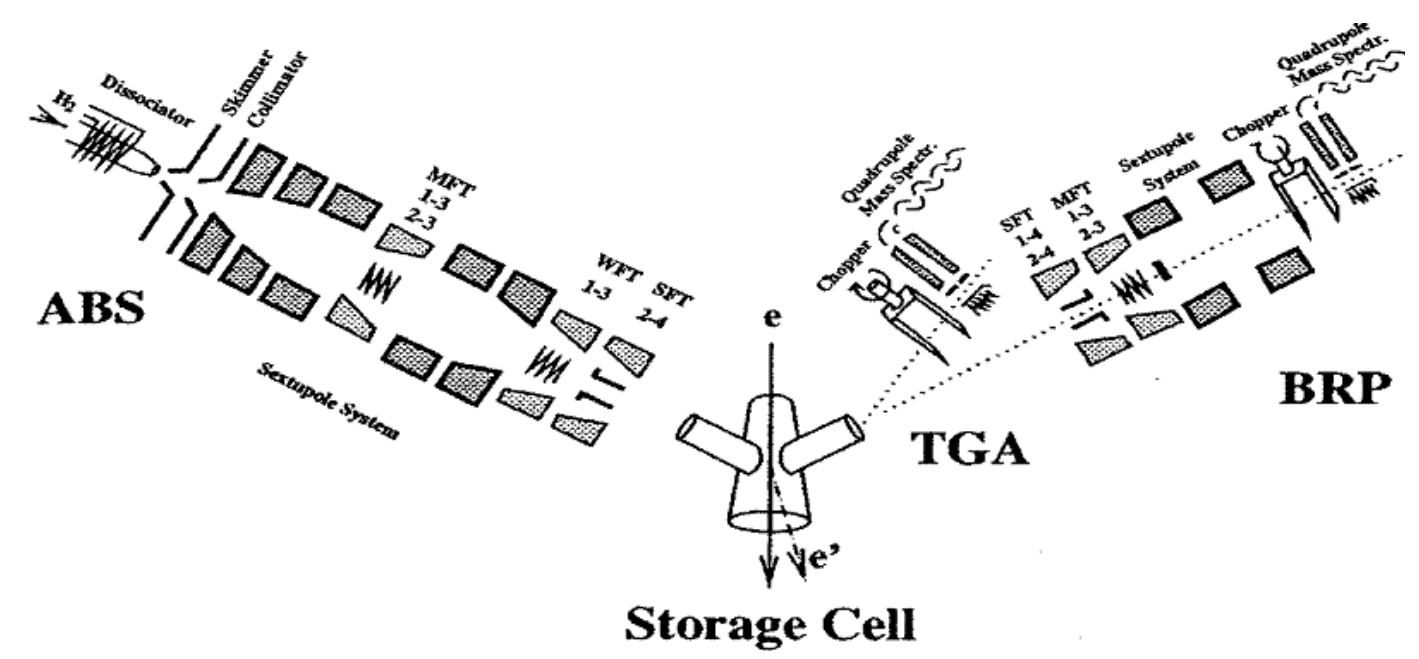

Fig. 1. Set-up of the HERMES polarized hydrogen target. The beam axis of ABS and BRP are tilted by $\pm 30^{\circ}$ with respect to the horizontal plane to avoid a direct beam into the BRP.

of the system of the colliding atoms, the spin-exchange process can be described by two different cross-section $\sigma^{+}$ and $\sigma^{-}$with the average value of $\sigma=\frac{1}{2}\left(\sigma^{+}+\sigma^{-}\right)$.

While theoretical values of the spin-exchange crosssection are available for a wide range of temperature extending from 10 to $1000 \mathrm{~K}$ [16], most of the measurements $[7,18-20]$ of this cross-section have been performed close to room temperature. Only one measurement of the spin-exchange cross-section exists up to now for the lower temperature range $77-363 \mathrm{~K}[21]$. In this region, $\sigma_{\text {se }}$ for $\mathrm{H}-\mathrm{H}$ collisions has been measured to be about $23.1 \times$ $10^{-16} \mathrm{~cm}^{2}$, which is in good agreement with Allison calculations [16]. The HERMES target [22] offered unique experimental conditions to extend the measurement of the spin-exchange collision cross-section down to the temperature of $40 \mathrm{~K}$. In particular, by making use of the polarized source, it was possible to inject different combinations of hydrogen hyperfine states in a storage cell of variable temperature. By varying the cell temperature and detecting the effect of the spin-exchange collisions on the hyperfine state populations by means of the target polarimeter, the spin-exchange collisions cross-section has been measured for the the first time in the temperature region $40-80 \mathrm{~K}$.

\section{The HERMES target}

The HERMES experiment at DESY (Hamburg) makes use of a polarized atomic hydrogen gas target to study the quark-gluon spin structure of the nucleons by deepinelastic lepton-nucleon scattering [23,24]. A schematic picture of the target is given in Figure 1. The atomic beam source (ABS) [25] injects a polarized beam of hydrogen atoms into the center of a Drifilm coated tubular storage cell [26] internal to the HERA electron ring. By using a storage cell, an areal nucleon density of up to $1.4 \times 10^{14}$ nucleons $/ \mathrm{cm}^{2}$ is obtained. The temperature of the storage cell can be continuosly varied between $40 \mathrm{~K}$ and $300 \mathrm{~K}$ by circulation of cold He gas. The target vacuum chamber containing the storage cell is surrounded by the external target magnet which provides a strong holding field to define the direction of polarization and to decouple the nuclear and electron spins. The nuclear polarization and the atomic fraction of the target gas are determined analyzing a sample beam diffusing through a side tube from the center of the cell to the Breit-Rabi polarimeter (BRP) [27] and to the target gas analyzer (TGA) [28], respectively, as it will be described in the next section.

\subsection{Breit-Rabi polarimeter and target gas analyzer}

The Breit-Rabi polarimeter precisely measures the relative populations of the hyperfine states of the atomic beam effusing from the sample tube. The nuclear and electronic polarizations of the atoms in a given magnetic field are linear functions of the hyperfine state population numbers $n_{i}$ and are calculated as described in reference [27].

The purpose of the target gas analyser is to measure the fraction of atoms and molecules of the gas sample. The TGA is integrated into the vacuum system of the BRP sextupole chamber. It consists of a pair of baffles, a chopper which periodically blocks the sample beam and allows subtraction of the residual gas signal and a beam detection system ${ }^{1}$. In contrast to the BRP where only hydrogen atoms are detected the TGA detects both atoms and molecules simultaneously.

To provide a relationship between the measured polarization $\left(P_{B R P}\right)$ and atomic fraction $\left(\alpha_{T G A}\right)$ for the sampled atoms and their average values in the target $\left(P_{a}\right.$ and $\left.\alpha_{r}\right)$, so called "sampling corrections" are introduced $\left(c_{B R P}\right.$ and $c_{T G A}$, which are evaluated through a careful analysis of the diffusion of atoms through the cell. Details of this analysis are reported elsewhere $[29,30]$. The average

1 The beam detection system contains a crossed beam ionizer in combination with a quadrupole mass spectrometer (Balzers QMA 430) and a channel electron multiplier for single ion detection. 
target polarization in the cell is defined by the following equation:

$$
P^{T}=\alpha_{0} \alpha_{r} P_{a}+\alpha_{0}\left(1-\alpha_{r}\right) \beta P_{a}
$$

where $\alpha_{0}$ represents the initial fraction of nucleons in atoms as injected by the ABS (or atomic fraction in absence of recombination), $\alpha_{r}$ denotes the fraction of nucleons in atoms surviving recombination during their way through the storage cell, $\beta=P_{m} / P_{a}$ [31] stands for the ratio of the polarization of the molecules $P_{m}$ produced by recombination relative to the polarization of the atoms $P_{a}$. The values of $\alpha_{r}$ and $P_{a}$ are related to the measured values from TGA and BRP by the relations:

$$
\begin{aligned}
& \alpha_{r}=c_{T G A} \alpha_{r}^{T G A}, \\
& P_{a}=c_{B R P} P_{a}^{B R P} .
\end{aligned}
$$

\subsection{The unpolarized gas feed system}

The unpolarized gas feed system (UGFS) [32] is required for the unpolarized physics program at the HERMES experiment. In the present work it has been used for the absolute calibration of the flux injected into the cell, in order to precisely determine the target density (see Sect. 4.2). The generated flux has been calibrated at the level of $2 \%$.

\section{Principle of measurement}

The HERMES hydrogen target makes use of an atomic beam source (ABS) in conjunction with a storage cell immersed in a strong magnetic holding field.

The atoms interacting with each other in the cell volume may experience spin exchange, causing a change in the hfs populations inside the storage cell. Angular momentum conservation forces the sum of the magnetic quantum numbers $m_{F}$ before and after the spin exchange collision to be the same:

$$
\sum m_{F}=\text { const. }
$$

Table 1 gives an overview of the allowed hyperfine transitions for any given pair of colliding hydrogen atoms being in one of four states $|1\rangle$ to $|4\rangle$, which correspond to the eigenstates of the hydrogen atoms in a ground state $1 \mathrm{~S}_{1 / 2}$ in a Breit-Rabi-basis. One can see that in case of the pure states $|1\rangle$ or $|3\rangle$ initially populating the gas no relaxation by means of spin exchange is possible. In general, a mixture of pure and mixed states is injected into the HERMES cell during the data taking. The important injection modes both for data taking and for the measurement described here are those with two states injected having a large positive $(|1\rangle+|4\rangle)$ or negative $(|2\rangle+|3\rangle)$ nuclear polarization $P_{z+}$ and $P_{z-}$, respectively (note that states $|2\rangle$ and $|4\rangle$ present high nuclear polarization only in a strong holding field).
Table 1. Pairs of hyperfine states of hydrogen for which spinexchange collisions are allowed by angular momentum conservation.

\begin{tabular}{ll}
\hline$\sum_{i} m_{F}$ & "Hydrogen hyperfine states" \\
\hline 2 & $|1\rangle,|1\rangle$ \\
1 & $|1\rangle,|2\rangle \leftrightarrow|1\rangle,|4\rangle$ \\
0 & $|1\rangle,|3\rangle \leftrightarrow|2\rangle,|4\rangle \leftrightarrow|2\rangle,|2\rangle \leftrightarrow|4\rangle,|4\rangle$ \\
-1 & $|2\rangle,|3\rangle \leftrightarrow|3\rangle,|4\rangle$ \\
-2 & $|3\rangle,|3\rangle$ \\
\hline
\end{tabular}

The effect of spin-exchange collisions on the hfs populations can be described by a tensor $M_{a b}^{c}$ which gives the probability to find an atom in state $|c\rangle$ after a collision of two atoms in the state $|a\rangle$ and $|b\rangle$ prior to the collision. The transition coefficients for a transition $|a b\rangle \rightarrow|c d\rangle$ can be explicitly calculated [33]. The total rate into state $|c\rangle$ is given by:

$$
\dot{n}_{c}=\dot{N}_{s e} \sum_{a, b} M_{a b}^{c} n_{a} n_{b}
$$

where $n_{i}$ is the occupation number of the hyperfine state $|i\rangle ; \dot{N}_{s e}=\langle n\rangle \sigma_{s e}\left\langle v_{r}\right\rangle[1]$ is the number of spin-exchange collisions per atom and unit time in a gas with the particle density $\langle n\rangle ;\left\langle v_{r}\right\rangle$ is the mean relative velocity calculated from Maxwell's velocity distribution, which for the case of two atoms of equal mass is $\left\langle v_{r}\right\rangle=\sqrt{2}\langle v\rangle$.

Spin-exchange collisions in an initially nuclear polarized gas (injected states $|1\rangle+|4\rangle$ or $|2\rangle+|3\rangle$ ) cause transfer of polarization from the nucleus to the electron resulting in a net depolarization of the gas. As derived in Section 5 (Eq. (23)), the relative nuclear depolarization caused by the spin-exchange collisions, for the case in which this is the dominant relaxation mechanism, can be described as:

$$
\delta P_{z}^{s e}=\frac{P_{z}^{i n j}-P_{z}}{P_{z}^{i n j}}=\frac{1}{2} \frac{1}{1+\tau_{s e} / \tau_{d}\left(1+x^{2}\right)},
$$

where $\tau_{d}$ is the diffusion time of hydrogen atoms within the storage cell, $\tau_{s e}$ is the mean time between two spinexchange collisions and $x$ is the ratio between the holding field and the critical field $B / B_{C}^{H}$ of hydrogen, $P_{z}^{i n j}$ is the initial nuclear polarization of the atoms. Taking into account that $1 / \tau_{s e}=\sqrt{2} \sigma_{s e}\langle v\rangle\langle n\rangle$ and $\tau_{d}=L_{d} /\langle v\rangle$, where $L_{d}$ is the diffusion length, one gets:

$$
\sigma_{s e}=\frac{\sqrt{2} \delta P_{z}^{s e}\left(1+x^{2}\right)}{L_{d}\langle n\rangle\left(1-2 \delta P_{z}^{s e}\right)}
$$

Thus by detecting the amount of the depolarization $\delta P_{z}^{s e}$ caused by spin-exchange collisions and measuring the target averaged density $\langle n\rangle$ it is possible to derive the spinexchange collision cross-section. 
Table 2. Pairs of hyperfine states injected by ABS into the cell and possible spin-exchange collisions allowed by angular momentum conservation. The definition of $\sigma^{+}$and $\sigma^{-}$is given in the text.

\begin{tabular}{clll}
\hline Injected & Collision & $\Delta P_{z}^{s e}$ & $\sigma_{\text {se }}$ \\
\hline \multirow{3}{*}{$|1\rangle,|4\rangle$} & $|1\rangle,|1\rangle$ & no & $\sigma^{+}$ \\
& $|1\rangle,|4\rangle$ & $\sqrt{ }$ & $\sigma^{-}$ \\
& $|4\rangle,|4\rangle$ & $\sqrt{ }$ & $\sigma^{+}$ \\
\hline \multirow{3}{*}{$|2\rangle,|3\rangle$} & $|3\rangle,|3\rangle$ & no & $\sigma^{+}$ \\
& $|2\rangle,|3\rangle$ & $\sqrt{ }$ & $\sigma^{-}$ \\
& $|2\rangle,|2\rangle$ & $\sqrt{ }$ & $\sigma^{+}$ \\
\hline
\end{tabular}

\section{Measurement}

Equation (10) has been employed to extract the spinexchange cross section. Three terms had to be determined: the amount of nuclear depolarization $\delta P_{z}^{s e}$, the averaged target density $\langle n\rangle$ and the diffusion length $L_{d}$ of the particles inside the cell.

\subsection{Measurement of $\delta \mathrm{P}_{\mathrm{z}}^{s e}$}

In the cell, relaxation due to spin-exchange is accompanied by relaxation due to wall collisions. The contribution $\delta P_{z}^{s e}$, coming from spin-exchange collisions, has been separated from the effect of wall collision via their different density dependence. In particular, while relaxation due to spin-exchange is density dependent, the contribution to depolarization by wall relaxation is not. The target density has been varied by changing the flux of the ABS. The amount of depolarization due to the spin-exchange process is extracted by interpolating the measured polarization as a function of the injected flux with a straight line and extrapolating to zero density. To measure $\delta P_{z}^{s e}$, pairs of hyperfine states $|1\rangle+|4\rangle\left(P_{z+}\right)$ and $|2\rangle+|3\rangle\left(P_{z-}\right)$ were injected into the cell (see Tab. 2).

Figure 2 shows the variation of nuclear polarization as a function of the ABS flux. As shown in Appendix, the polarization loss caused by spin-exchange collision relative to the injected polarization can be written in the following way:

$$
\delta P_{z}^{s e}=\frac{\left(P_{z}\right)_{0}-\left(P_{z}\right)_{1}}{P_{z}^{i n j}},
$$

where $\left(P_{z}\right)_{1}$ is the atomic polarization measured at nominal density, and the $\left(P_{z}\right)_{0}$ is the extrapolated polarization for zero density. Note that $\left(P_{z}\right)_{0}$ is different from $P_{z}^{i n j}$ because of the effect of wall relaxation.

The injected nuclear polarization $P_{z}^{i n j}$, i.e. the nuclear polarization in absence of depolarization, for each above mentioned ABS operation has been calculated by using the transmission probabilities of the sextupole system and the efficiencies of the adiabatic transition units in the ABS [25]. This results in injected nuclear polarizations $P_{z+}^{i n j}=0.973 \pm 0.010$ and $P_{z-}^{i n j}=-0.974 \pm 0.010$, respectively.
Example of measurement for the evaluation of $\delta P_{z}^{s e}$
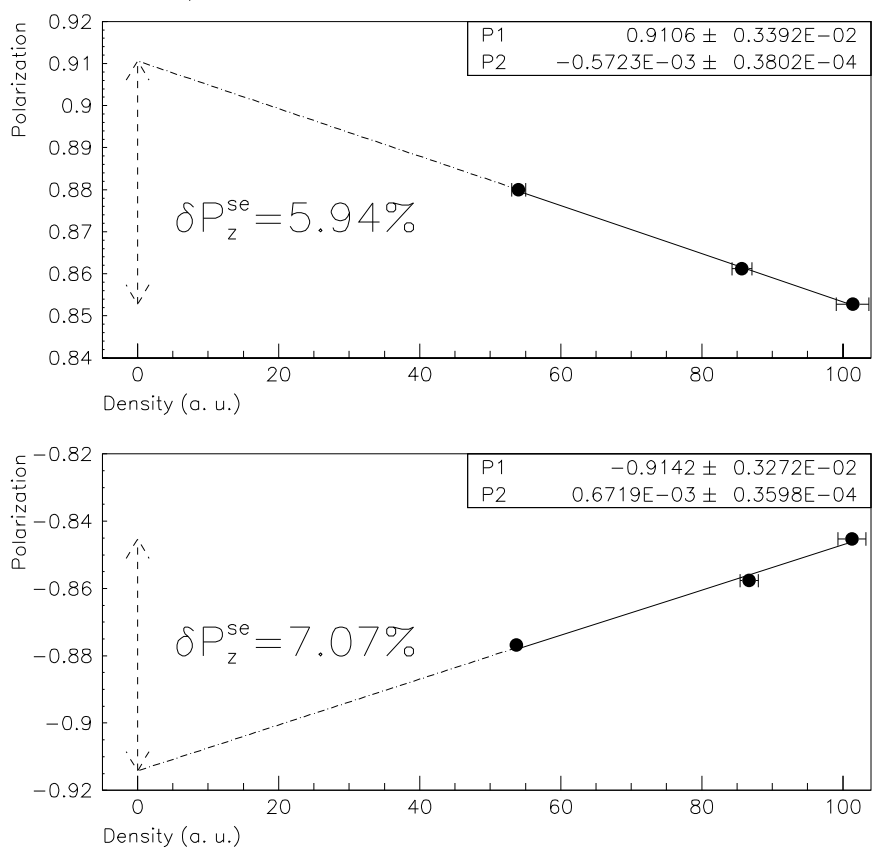

Fig. 2. Density dependence of the polarization measured by BRP for two different set of injected hyperfine states. The upper (lower) plot corresponds to the set of injected states with polaization $P_{z+}\left(P_{z-}\right)$. For details see text.

Table 3. Spin-exchange cross-sections for different sets of measurements. The errors presented are the total statistical plus systematic errors.

\begin{tabular}{|c|c|c|c|c|}
\hline Temp. & $\begin{array}{l}\text { First set } \\
\sigma_{s e} \\
10^{-16} \mathrm{~cm}^{2}\end{array}$ & $\begin{array}{l}\text { Second set } \\
\sigma_{s e} \\
10^{-16} \mathrm{~cm}^{2}\end{array}$ & $\begin{array}{l}\text { Third set } \\
\sigma_{s e} \\
10^{-16} \mathrm{~cm}^{2}\end{array}$ & $\begin{array}{l}\text { Average } \\
\sigma_{s e} \\
10^{-16} \mathrm{~cm}^{2}\end{array}$ \\
\hline 40 & 2 & 19 . & 20. & 19. \\
\hline 60 & $20.1 \pm 1.0$ & $19.9 \pm 1.4$ & no & $20.1 \pm 2.2$ \\
\hline 80 & $24.5 \pm 1.4$ & $24.0 \pm 1.2$ & no & $24.2 \pm 2.2$ \\
\hline 100 & $22.5 \pm 2.3$ & $26.9 \pm 1.9$ & no & $25.7 \pm 3.6$ \\
\hline
\end{tabular}

Depolarization has been measured for four different temperatures $(40 \mathrm{~K}, 60 \mathrm{~K}, 80 \mathrm{~K}$ and $100 \mathrm{~K})$. Two sets of measurements have been taken for all four temperatures (see Tab. 3). An additional measurement has been taken at $40 \mathrm{~K}$.

As the change in the temperature affects the recombination inside the cell, all the measurements have been corrected by the measured atomic fraction $\alpha_{r}^{T G A}[22]$ before the fit procedure.

\subsection{Measurement of the target density $\langle\mathbf{n}\rangle$}

In order to derive an absolute value for the spin-exchange cross-section, the target density has to be known. To this aim a precise measurement of the ABS flux has been performed. Two units were used for this purpose, the UGFS, which allows to inject into the storage cell a known flux, 


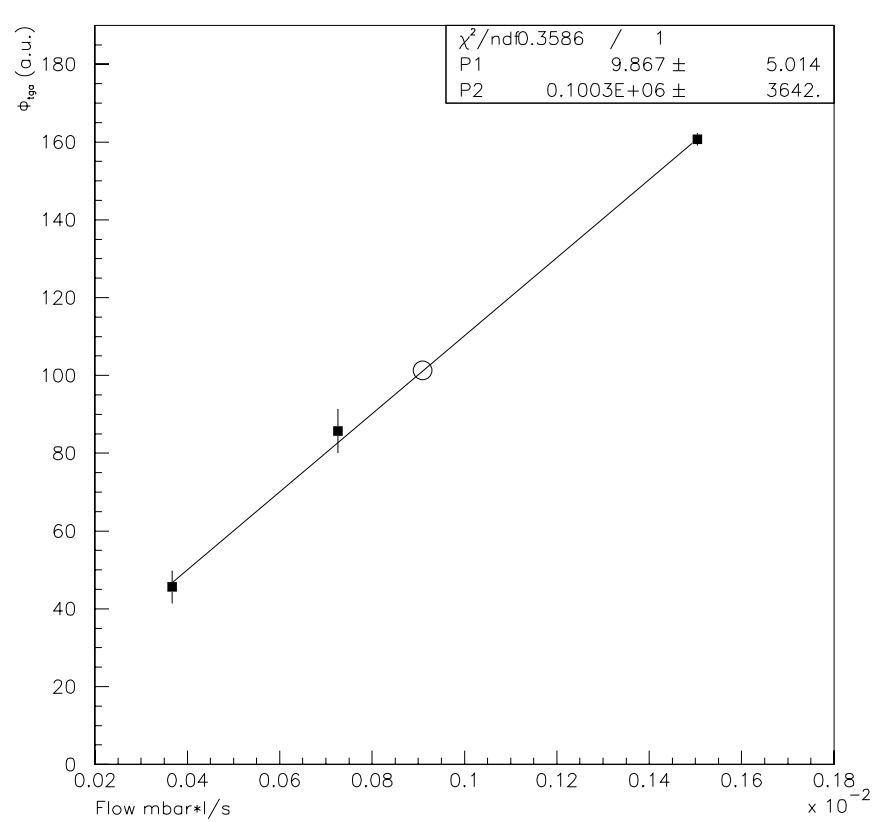

Fig. 3. The TGA count rates (in a.u.) as a function of UGFS flow (in mbar $\times 1 / \mathrm{s}$ ) with the point (open circle) corresponding to the ABS flux. The error bars are only statistical. The error of the ABS point is smaller than its size.

and the TGA, the total rate of which (i.e. hydrogen atoms plus molecules) is proportional to the target density.

The value of the ABS flux have been derived by comparison to precisely known $\mathrm{H}_{2}$ molecular fluxes from the UGFS. In Figure 3 the measured TGA count rates vs the flow coming from the UGFS are plotted together with a linear fit. The open circle refers to the ABS flux obtained by projection of the measured TGA count rate to this line. The resulting ABS flux $\Phi_{A B S}$ calculated from the TGA count rates is equal to $(9.10 \pm 0.13) \times 10^{-4} \mathrm{mbar} \times 1 / \mathrm{s}$ or $\Phi_{A B S}=(6.57 \pm 0.09) \times 10^{16}$ atoms $/ \mathrm{s}$.

To convert the measured flux into a density value, the cell conductance is required. For the HERMES geometry a Monte Carlo program [34] was used. The results of the simulation for the total conductance is: $C_{T}=$ $9110 \pm 5 \mathrm{~cm}^{3} / \mathrm{s}$. As the cell conductance has been evaluated at $100 \mathrm{~K}$, to obtain the value of the target density at different temperatures, one has to scale the given conductance according to $C_{T}=C_{100} \sqrt{T / 100 \mathrm{~K}}$. The average density in the cell is then given by

$$
\langle n\rangle=\frac{\Phi_{A B S}}{2 C_{T}} .
$$

\subsection{Derivation of the diffusion length $L_{d}$}

The same Monte Carlo program [34], used for the evaluation of the total conductance of the cell, has been employed to determine the diffusion length $L_{d}$ of the particles inside the cell (see Eq. (10)). The diffusion length is defined as the integral path passed by a single particle through the cell geometry [22], starting at the injection tube and ending in the extension tube. It was proven

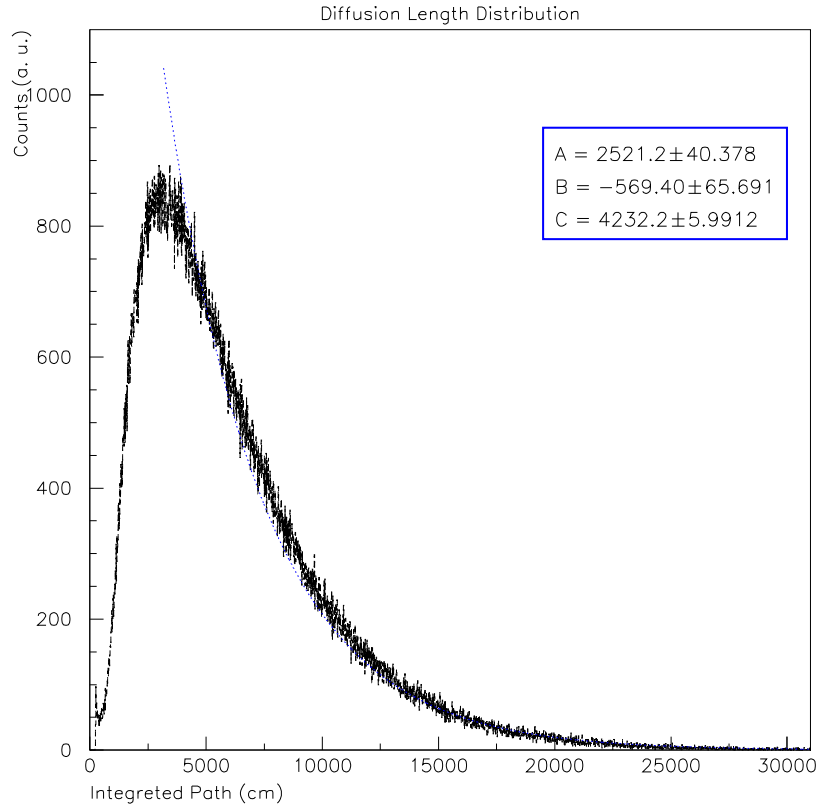

Fig. 4. (Color online) Distribution of the diffusion length obtained by Monte Carlo simulation generating $10^{7}$ particles. For more details see text.

in [29] that the diffusion length extracted by fitting the diffusion length distribution, obtained from the intensity change of the measured BRP signal, for a sample of deuterium gas differs by less than $0.2 \%$ from the diffusion length derived from the Monte Carlo simulation.

To obtain the distribution shown in Figure 4, $10^{7}$ particles have been generated and tracked along the cell geometry. Only $5.43 \%$ of the generated particles passed through the extension tube. Left of the maximum, the distribution reflects the Maxwell-Boltzmann thermal distribution of the particles. The part of the plot of interest for this calculation, i.e. the diffusive part of the distribution, is the one going from the maximum of the distribution to the end. In order to extract the value of the diffusion length, a fit was performed on the last part of the distribution, shown in Figure 4 as dotted line, with the following fit function:

$$
y=A e^{\frac{-x+B}{C}},
$$

where the coefficients $A, B$ and $C$ are the normalization factor, the offset and the diffusion length, respectively. From the fit a value for the diffusion length of $4232.20 \pm 5.99 \mathrm{~cm}$ has been determined.

\subsection{Results}

The measurements of the relative loss of the nuclear polarization $\delta P_{\text {se }}$ and the target averaged density $\langle n\rangle$ enable to calculate the spin-exchange cross-section from equation (10). The results are presented in Table 3 . The reported errors represent the total uncertainties. As the polarization loss $\delta P_{s e}$ is independent from the sign of the nuclear polarization the half difference between the values of cross-section obtained injecting $P_{z}^{+}$and $P_{z}^{-}$is assigned 
as systematic uncertainty. The contributions from the other sources to the total uncertainty are associated with the density measurement and the fit procedure amounting to $5 \%$ and $0.5 \%$, respectively. The final value of the spin-exchange cross-section is obtained by the weighted average of the set of measurements. The temperature dependence of the hydrogen-hydrogen spin-exchange crosssection is shown in the upper plot of Figure 5 together with three different theoretical predictions from $[1,14,16]$. The middle dotted line represents the theoretical values of $\sigma$ derived from $\sigma^{+}$and $\sigma^{-}$calculated by Allison [16]. The solid lines specify the $10 \%$ uncertainty limits on these theoretical values. Our results agree well with the Allison's prediction. In the lower plot of Figure 5 the experimental results on the spin-exchange cross-section (closed squares) vs. temperature are shown together with the other experimental existing data [21] (triangles). Experimental data and calculations agree satisfactorily within the errors in the extended range of temperature.

\section{Conclusions}

The hydrogen spin-exchange collision cross-section has been measured for the first time in the temperature range 40-100 K using the polarized hydrogen gas target of the HERMES experiment at DESY. The experimental results have been compared with three different theoretical calculations, all predicting a strong temperature dependence below $100 \mathrm{~K}$. The measured cross-section agrees quite well with the theoretical calculation performed by Allison. The only other data of the spin-exchange cross-section for hydrogen at low temperature exists for the temperature range $80-300 \mathrm{~K}$. The new experimental data agree with a previous measurement in the overlapping temperature region $(80-100 \mathrm{~K})$ and seem to hint a slightly increasing behaviour of the cross-section with temperature in the region 50-80 K. This tendency is in agreement with an early estimation by Allison [16], while it seems to contradict the decreasing behaviour predicted by more recent theoretical calculations by Swenson et al. [14], Cole and Olson [35] and Braga and Varandas [36]. Additional measurement in the critical region $0-50 \mathrm{~K}$ would be highly desirable to clearly distinguish between the two scenarios. These could not be performed with the experimental apparatus described in the paper, the main limit to the achievement of lower temperature coming from the radiation heat load on the cell walls by the vacuum chamber.

\section{Appendix: The transformed master equation for hydrogen}

The relaxation process due to the two-body spin-exchange collisions of atoms is not the only process taking place inside the HERMES target. The atoms injected by atomic beam source experience some hundreds of wall collisions during their diffusion process through the storage cell. This can be described by means of transition probabilities $W_{a c}$ which represents the probability to find an atom
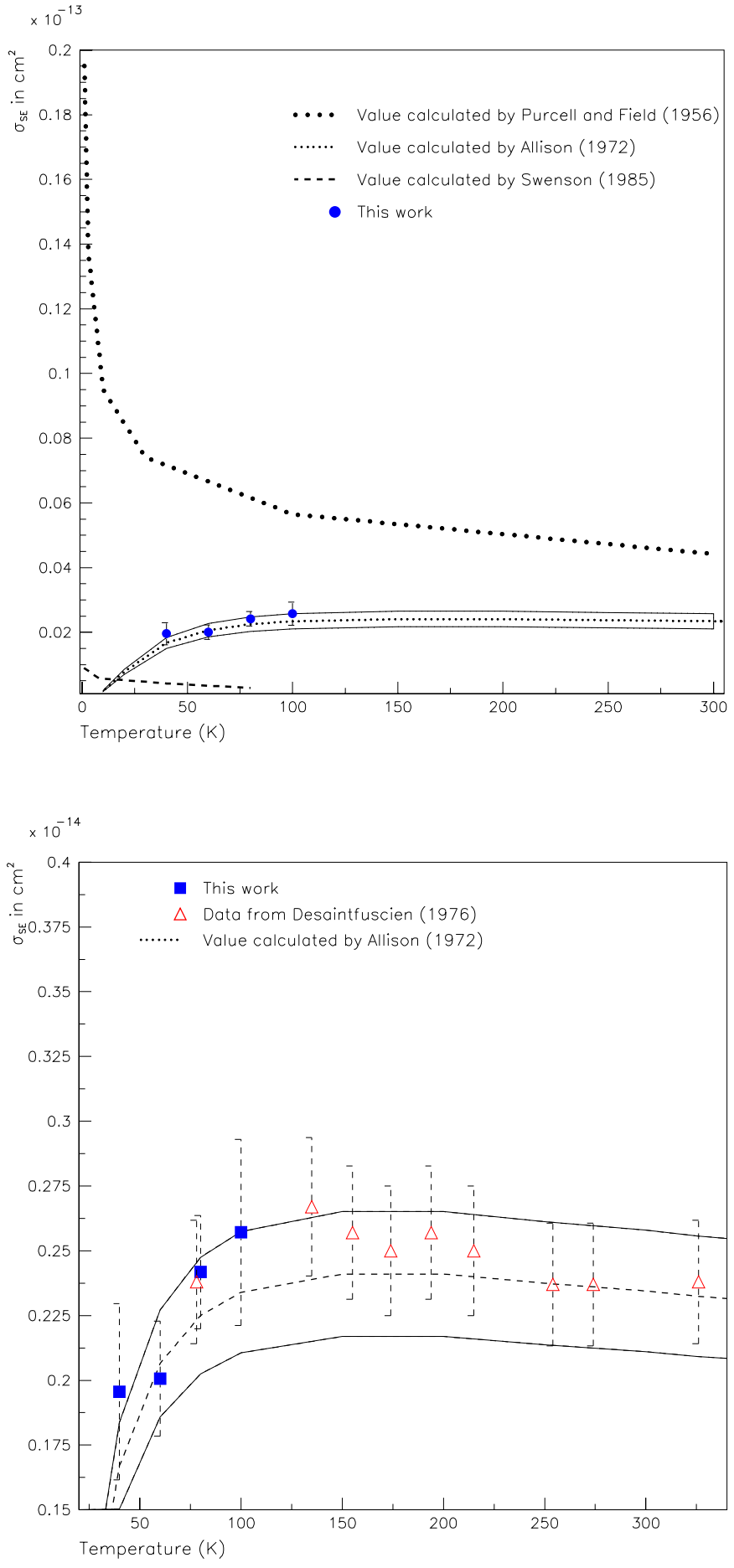

Fig. 5. (Color online) Overview of the experimental results and the theoretical predictions from $[1,14,16,21]$. Upper plot: the middle dotted line corresponds to the theoretical values of $\sigma$ derived from $\sigma^{+}$and $\sigma^{-}$calculated by Allison [16] and the solid lines specify the $10 \%$ uncertainty limits on these theoretical values. The squared represent the results from the present work. Lower plot: the dashed line is again from Allison [16] and the solid lines again show the $10 \%$ systematic uncertainty of this calculation. The result obtained with HERMES target are shown as squares. The triangles are from [21]. 
in state $|c\rangle$ that have been in state $|a\rangle$ prior to the wall collision. Since the atoms are constantly exposed to both above mentioned interactions, an appropriate description is given by so called master equation $[29,34]$ :

$$
\begin{aligned}
\dot{n_{c}}= & \frac{1}{\tau_{d}}\left(n_{c}^{i n j}-n_{c}\right)+\frac{\tau_{d}}{\tau_{f}} \sum_{a} R_{c a} n_{a} \\
& +\frac{\tau_{d}}{\tau_{s e}} \sum_{a, b} M_{a b}^{c} n_{a} n_{b},
\end{aligned}
$$

where the matrix $R_{c a}$ is related to the transition probability per wall collision in the following way [37]:

$$
R_{c a}=W_{a c}-\delta_{c a} \sum_{b} W_{b c}
$$

with the Kronecker symbol $\delta_{c a}$. First part in right side of equation (14) represents the incoming and outgoing flux, the second the wall relaxation and the last term the spin exchange relaxation. The effect of recombination, which is negligible during the optimal operation of the HERMES target, is not included in this formula. The three time constants in equation (14) are: $\tau_{d}$ the mean occupation time (the diffusion time) of atoms within the storage cell, $\tau_{f}$ the mean time of flight between two wall collisions and $\tau_{\text {se }}$ the mean time between two spin-exchange collisions. For times that are large compared to $\tau_{d}$ the hyperfine population converges toward a dynamical equilibrium (steady state). The linear algebraic equation system (14) in the steady state has been solved directly in the case of hydrogen [34].

A fairly simple set of equation to describe of hydrogen can be obtained by a Hadamard transformation of the master equation (14). The transformed variable are given by:

$$
\left(\begin{array}{l}
\mathcal{W}_{0} \\
\mathcal{W}_{e} \\
\mathcal{W}_{z} \\
\mathcal{W}_{p}
\end{array}\right)=\frac{1}{2}\left(\begin{array}{rrrr}
1 & 1 & 1 & 1 \\
1 & 1 & -1 & -1 \\
1 & -1 & -1 & 1 \\
1 & -1 & 1 & -1
\end{array}\right)\left(\begin{array}{l}
n_{1} \\
n_{2} \\
n_{3} \\
n_{4}
\end{array}\right)
$$

The master equation is then:

$$
\begin{aligned}
0 & =\mathcal{W}_{c}^{i n j}-\mathcal{W}_{c}+\frac{\tau_{d}}{\tau_{f}} \tilde{R}_{c a} \mathcal{W}_{a}+\frac{\tau_{d}}{\tau_{s e}} \tilde{M}_{a b}^{c} \mathcal{W}_{a} \mathcal{W}_{b} \\
\tilde{R}_{c a} & =T_{c b} R_{b d} T_{d a} \\
\tilde{M}_{a b}^{c} & =T_{c d} M_{e f}^{d} T_{e b} T_{f a} .
\end{aligned}
$$

Using the following shortcuts

$$
\begin{array}{ll}
a=\left\langle W_{12}\right\rangle & b=\left\langle W_{14}\right\rangle \\
c=\left\langle W_{23}\right\rangle & d=\left\langle W_{34}\right\rangle \\
e=\left\langle W_{24}\right\rangle & f=\frac{\sin ^{2} 2 \theta}{2},
\end{array}
$$

the resulting transformed master equations are:

$$
\begin{aligned}
0= & \mathcal{W}_{e}^{i n j}-\mathcal{W}_{e}+\frac{\tau_{d}}{\tau_{f}}\left\{-(b+c+e) \mathcal{W}_{e}+e \mathcal{W}_{z}\right. \\
& \left.+(c-b) \mathcal{W}_{p}\right\}+\frac{\tau_{d}}{\tau_{s e}} f\left(\mathcal{W}_{z}-\mathcal{W}_{e}\right) \\
0= & \mathcal{W}_{z}^{i n j}-\mathcal{W}_{z}+\frac{\tau_{d}}{\tau_{f}}\left\{-(a+d+e) \mathcal{W}_{z}+e \mathcal{W}_{e}\right. \\
& \left.+(d-a) \mathcal{W}_{p}\right\}+\frac{\tau_{d}}{\tau_{s e}} f\left(\mathcal{W}_{e}-\mathcal{W}_{z}\right) \\
0= & \mathcal{W}_{p}^{i n j}-\mathcal{W}_{p}+\frac{\tau_{d}}{\tau_{f}}\left\{(c-b) \mathcal{W}_{e}+(d-a) \mathcal{W}_{z}\right. \\
& \left.-(a+b+c+d) \mathcal{W}_{p}\right\} \\
& +\frac{\tau_{d}}{\tau_{s e}}\left\{2 \mathcal{W}_{e} \mathcal{W}_{z}-\mathcal{W}_{p}+f\left(\mathcal{W}_{e}-\mathcal{W}_{z}\right)^{2}\right\}
\end{aligned}
$$

If the wall depolarization is neglected, i.e., $a=b=c=$ $d=e=0$, equation (19) yields:

$$
\begin{aligned}
& \left.0=\mathcal{W}_{e}^{i n j}-\mathcal{W}_{e}+\frac{\tau_{d}}{\tau_{s e}} f\left(\mathcal{W}_{z}-\mathcal{W}_{e}\right)\right) \\
& \left.0=\mathcal{W}_{z}^{i n j}-\mathcal{W}_{z}+\frac{\tau_{d}}{\tau_{s e}} f\left(\mathcal{W}_{e}-\mathcal{W}_{z}\right)\right) \\
& 0=\mathcal{W}_{p}^{i n j}-\mathcal{W}_{p}+\frac{\tau_{d}}{\tau_{s e}}\left\{2 \mathcal{W}_{e} \mathcal{W}_{z}-\mathcal{W}_{p}+f\left(\mathcal{W}_{e}-\mathcal{W}_{z}\right)^{2}\right\}
\end{aligned}
$$

These equations can be solved directly:

$$
\begin{aligned}
& \mathcal{W}_{e}=\mathcal{W}_{e}^{i n j}-\frac{1}{2} \frac{2 \frac{\tau_{d}}{\tau_{s e}} f}{1+2 \frac{\tau_{d}}{\tau_{s e}} f}\left(\mathcal{W}_{e}^{i n j}-\mathcal{W}_{z}^{i n j}\right) \\
& \mathcal{W}_{z}=\mathcal{W}_{z}^{i n j}-\frac{1}{2} \frac{2 \frac{\tau_{d}}{\tau_{s e}} f}{1+2 \frac{\tau_{d}}{\tau_{s e}} f}\left(\mathcal{W}_{z}^{i n j}-\mathcal{W}_{e}^{i n j}\right) .
\end{aligned}
$$

Due to the linearity and symmetry of equations (21) and (22), they are also valid for $P_{e}$ and $P_{z}$ correspondingly. In case of hydrogen two state injection mode, where either $\left(P_{e}\right)_{0}$ or $\left(P_{z}\right)_{0}$ are close to zero, the relative polarization loss $\delta P_{z}^{s e}$ by spin exchange collisions is:

$$
\begin{aligned}
\delta P_{z}^{s e} \simeq \frac{P^{i n j}-P}{P^{i n j}} & =\frac{1}{2} \frac{2 \frac{\tau_{d}}{\tau_{s e}} f}{1+2 \frac{\tau_{d}}{\tau_{s e}} f} \\
& =\frac{1}{2} \frac{1}{1+\frac{\tau_{s e}}{\tau_{d}}\left(1+x^{2}\right)},
\end{aligned}
$$

where $\left(1+x^{2}\right) \simeq 1 / \sin ^{2} 2 \theta[37]$.

\section{References}

1. E.M. Purcell, G.B. Field, Astrophys. J. 124, 524 (1956)

2. A. Dalgarno, Proc. R. Soc. A 262, 132 (1961)

3. A. Dalgarno, R.J.W. Henry, Proc. Phys. Soc. Lond. 83, 157 (1964)

4. A. Dalgarno, Rev. Mod. Phys. 39, 850 (1967)

5. J.P. Wittke, R.H. Dicke, Phys. Rev. 103, 620 (1956) 
6. A.F. Hildebrandt, F.B. Booth, C.A. Barth, J. Chem. Phys. 31, 273 (1959)

7. R.M. Mazo, J. Chem. Phys. 34, 169 (1961)

8. P.L. Bender, Phys. Rev. 132, 2154 (1963)

9. S.B. Crampton, H.T.M. Wang, Phys. Rev. A 12, 1305 (1975)

10. S.B. Crampton, Phys. Rev. 158, 57 (1967)

11. L.C. Balling, R.J. Hanson, F.M. Pipkin, Phys. Rev. A 607, $133(1964)$

12. F.J. Smith, Planet. Space Sci. 11, 126 (1963)

13. J.O. Hirschefelder, C.F. Curtiss, R.B. Bird, Molecular Theory of Gases and Liquids (John Wiley \& Sons, New York, 1954)

14. D.R. Swenson, D. Tupa, L.W. Anderson, J. Phys. B: At. Mol. Opt. Phys. 18, 4433 (1985)

15. T.E. Sharp, At. Data 2, 119 (1971)

16. A.C. Allison, Phys. Rev. A 5, 2695 (1972)

17. W. Kolos, L. Wolniewicz, J. Chem. Phys. 43, 2429 (1965)

18. H. Hellwig, Phys. Rev. 166, 4 (1968)

19. C. Audoin, Phys. Lett. A 28, 372 (1968)

20. J. Vanier, R. Brousseau, R. Larouche, Can. J. Phys. 51, 1901 (1973)

21. M. Desaintfuscien, C. Audoin, Phys. Rev. A 13, 2070 (1976)

22. A. Airapetian et al., Nucl. Instrum. Meth. A 540, 68 (2005)
23. HERMES-Collaboration, HERMES-Proposal (1990)

24. HERMES-Collaboration, HERMES Technical Design Report (1993)

25. A. Nass et al., Nucl. Instrum. Meth. A 505, 633 (2003)

26. C. Baumgarten et al., Nucl. Instrum. Meth. A 496, 277 (2003)

27. C. Baumgarten et al., Nucl. Instrum. Meth. A 482, 606 (2002)

28. C. Baumgarten et al., Nucl. Instrum. Meth. A 508, 268 (2003)

29. C. Baumgarten, Ph.D. thesis, University of Munich, Germany, DESY-THESIS-2000-038 (2006)

30. C. Baumgarten et al., Eur. Phys. J. D 18, 37 (2002)

31. A. Airapetian et al., Eur. Phys. J. D 29, 21 (2004)

32. M. Capiluppi, Ph.D. thesis, Universitati'degli Studi di Ferrara (2006)

33. T. Walker, L.W. Anderson, Nucl. Instrum. Meth. A 334, $313(1993)$

34. B. Braun, Ph.D. thesis MPIH-V25, Ludwig Maximilians-University, Munich, Germany (1995)

35. H.R. Cole, R.E. Olson, Phys. Rev. A 31, 2137 (1985)

36. J.P. Braga, A.J.C. Varandas, J. Phys. B: At. Mol. Opt. Phys. 23, 3113 (1990)

37. A. Abragam, The Principle of Nuclear Magnetism (Oxford University Press, 1983) 